\title{
Diagnóstico de los PRODUCTOS TURísticos
}

\author{
DIAGNOSE OF TOURISTIC PRODUCTS
}

Ph.D. Carlos Funcia Morán

Universidad Tecnológica Israel cfuncia@uisrael.edu.ec

Fecha de recepción: 17/05/2014

Fecha de aceptación: 18/06/2014

\section{Resumen}

Para lograr ofertar productos turísticos pertinentes, competitivos y sostenibles, se hace necesario partir de un diagnóstico que tenga en cuenta el carácter holístico, sistemático y cíclico del proceso de la actividad turística en que se enmarca el producto, con vista a obtener los conocimientos sobre las dificultades y sus causas en las ofertas, los logros alcanzados y potencialidades existentes. El presente artículo tiene como propósito dar a conocer los aspectos fundamentales a tener en cuenta para la realización del diagnóstico, como punto de partida para el desarrollo de la mejora o diseño de productos turísticos que respondan a demandas del mercado y como consecuencia permita contribuir al al éxito deseado según la misión y visión de las empresas.

Palabras clave: Turismo, Diagnóstico, Empresas, Competitividad, Sostenibilidad 


\begin{abstract}
To be able to offer qualified, competitive and long term tourist products, it is necessary to start from a diagnostic analysis that takes into account the holistic, systemic and cyclic framework of the end product tourist activity, geared towards gaining knowledge of the difficulties and causes of the offers, success stories and existing potentialities. The purpose of this article is to show the fundamental aspects to take into account when preparing a diagnostic analysis, to be used as the starting point for the development of improvements or design of tourist products that addresses the market demand as a consequence adds to the desired level of success in accordance to the mission and vision of the companies.
\end{abstract}

Keywords: Tourism, Diagnose, Enterprises, Competitivity, Sustainability 


\section{Diagnóstico de los productos turísticos}

Teniendo en cuenta el objeto de estudio de la actividad turística, dando como el proceso administrativo de la relación entre las ofertas y las demandas de productos turísticos, en los momentos actuales a los gestores de dicho proceso la sociedad les impone como necesidad social (demandas del mercado) un desempeño laboral eficiente orientado a la constante renovación de las ofertas como resultado de mejora o diseño de nuevos productos que según el contexto de actuación de la empresa y/o destino, permitan la obtención de productos pertinentes, competitivos y sostenibles.

Hoy por hoy, cuando en la inmensa mayoría de los casos del desarrollo de la actividad turística en una empresa y/o destino, la oferta de los productos turísticos es mayor que las demanda del mercado, resulta casi imprescindible para el logro de la mejora o diseño de los nuevos productos, con vista a aumentar las utilidades mediante la satisfacción de los clientes, partir de la obtención de conocimientos de las dificultades y sus causas en ofertas, los logros alcanzados y las potencialidades existentes.

Para el logro del conocimiento previo necesario, como punto de partida de la mejora o diseño de productos, es necesario tener en cuenta el carácter holístico, sistemático y cíclico del proceso de la actividad turística en que se enmarca el producto, así como las relaciones causa-efecto que se ponen de manifiesto.

Sin embargo, en aras del cumplimiento del reto impuesto a los gestores del proceso de mejora o diseño de productos turísticos, no en pocos casos se observa un modo de actuación en los mismos que no conduce a la dirección y desarrollo de un proceso óptimo que permita lograr los objetivos de una forma más eficiente y eficaz.

Es por tal sentido que el presente artículo tiene como propósito dar a conocer los aspectos fundamentales a tener en cuenta para la realización del diagnóstico, como punto de partida para el desarrollo de la mejora o diseño de productos turísticos que respondan a las demandas del mercado y como consecuencia permita contribuir al éxito deseado según la misión y visión de las empresas.

Para el desarrollo del diagnóstico y la comprensión del papel y el rol del mismo, se debe partir de su definición y en este orden se aporta la dada por el autor del presente artículo (Funcia, 2012) que plantea: 
Es el punto de partida para la gestión del proceso de mejora y/o diseño de productos turísticos, sentando las bases para la toma de decisiones en cada circunstancia, en correspondencia con el contexto donde se gestiona dicho proceso y el objetivo propuesto, a partir de un análisis profundo de la situación actual, mediante el empleo de métodos e instrumentos que permiten conocer los logros alcanzados, identificar las dificultades y oportunidades, así como las causas que limitan el logro del objetivo y los elementos que pueden acelerar el cumplimiento del mismo. (Funcia, 2012)

A partir de la definición anterior, a la hora de la realización de un diagnóstico hay que precisar el objetivo que se persigue con el mismo, como puede ser de forma parcial a una parte componente del producto o de forma integral teniendo en cuenta las relaciones causales que se ponen de manifiesto en su desarrollo dentro de la actividad turística, así como los elementos a diagnosticar y la selección de los métodos pertinentes en correspondencia con el objetivo propuesto.

De la precisión del objetivo del diagnóstico depende la selección de los elementos a diagnosticar, es decir, el ¿qué diagnosticar?

Para la determinación de los elementos a diagnosticar han sido valorados entre otros trabajos que abordan el diseño de productos turísticos (Beltrán et al., 2002), (SECTUR de México, 1998), (Mazur, Glenn H., 2002), (Perelló, 2001), (Martín, 2003). Los que nos permiten dar a conocer a continuación los elementos fundamentales sobre ¿qué diagnosticar?, para darle cumplimiento a un objetivo integral teniendo en cuenta el carácter holístico, sintético y cíclico del proceso de la actividad turística donde se desarrolla el producto.

Para la determinación del ¿qué diagnosticar?, se deben valorar los elementos fundamentales de tipo endógenos y exógenos que en sus relaciones causales influyen en la calidad de las ofertas de los productos.

Los sustentos teóricos principales a considerar en la mejora o diseño de los productos es por un lado la relación entre las oportunidades del mercado y las potencialidades existentes para la mejora o diseño de productos y esencialmente dentro de ellas las relacionadas con las ofertas de ¿qué percibir? y ¿qué hacer?, en correspondencia con las necesidades, intereses y deseos de los clientes (turistas) actuales y potenciales que constituyen demandas del mercado y por otro lado en sentido general la teoría del Marketing Turístico.

Dada la condición de que las ofertas resultan mayores que las demandas del mercado, los competidores son los vendedores de los productos iguales o similares a los que se gestionan dentro de la mejora o diseño, aspecto este que se presupone la necesidad de tener en cuenta a la hora de realizar un diagnóstico integra, valorar las ofertas de los competidores, así como de sus infraestructuras y servicios generales y de la organización y gestión del turismo con que cuentan para proporcionar resultados satisfactorios en la comercialización de sus productos. 
En resumen, la variable fundamental a considerar en el diagnóstico de los productos turísticos desde el punto de vista exógeno en la gestión de la mejora o diseño de los productos de una empresa y/o destino, lo constituye la determinación de las oportunidades del mercado y los elementos principales a considerar para ella son: la determinación de las demandas del mercado y la valoración de las características de los productos de los competidores, así como de las características de las infraestructuras y servicios generales y de organización y de gestión del turismo con que cuentan los competidores para el desarrollo de las ofertas de sus productos.

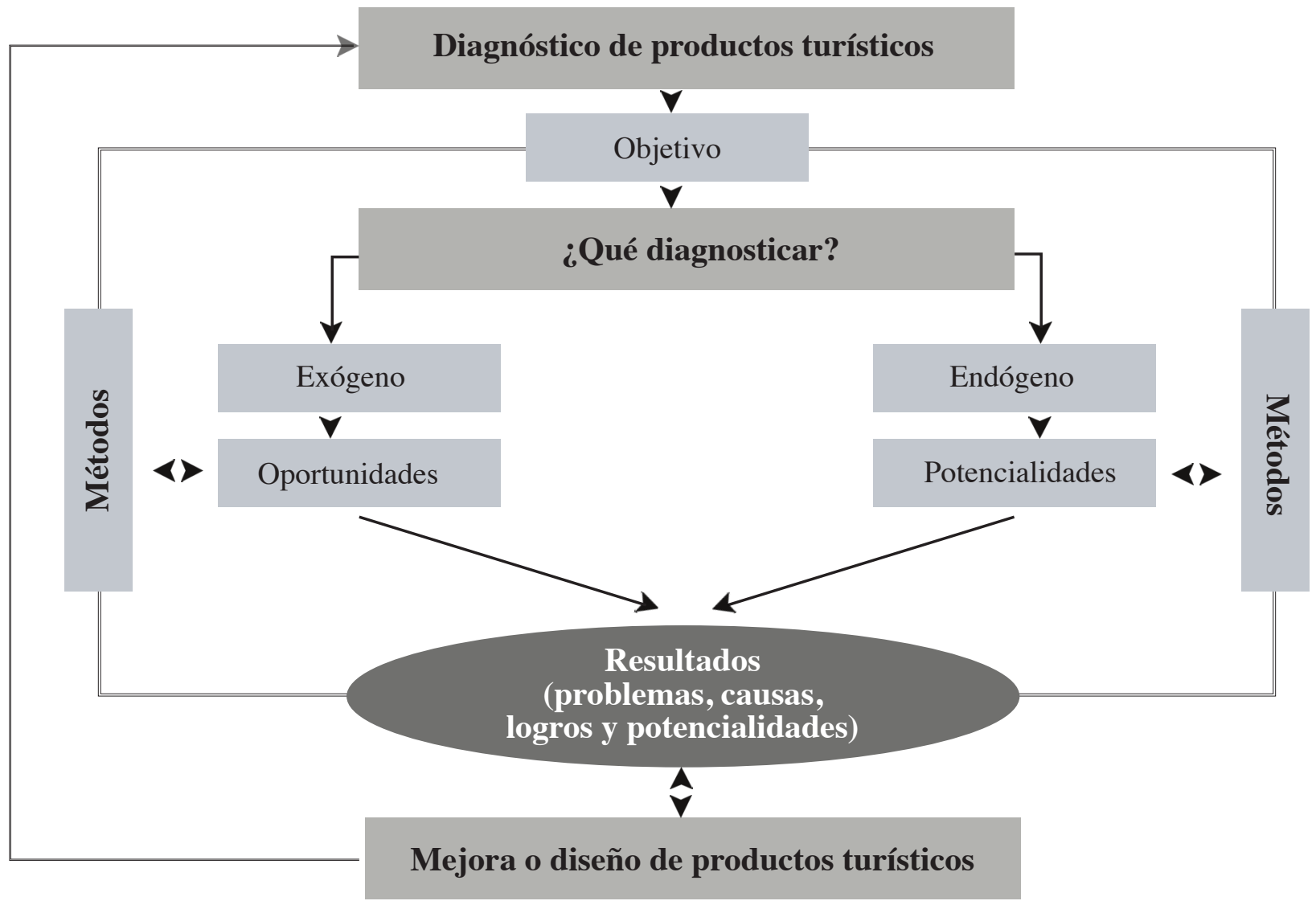


Desde el punto de vista endógeno en la gestión de la mejora o diseño de los productos en una empresa y/o destino, la variable principal a considerar en el diagnóstico es la determinación de las potencialidades existentes que puedan dar una respuesta pertinente a las oportunidades del mercado. Los elementos fundamentales a considerarse en este sentido son: la determinación de los recursos turísticos que puedan ser tenidos en cuenta como atractivos, la valoración general de los productos que se ofertan por parte de la empresa y/o destino donde se gestiona la mejora o diseño de los productos, así como de las características de las infraestructuras y servicios generales, de organización y de gestión del turismo con que cuenta la empresa y/o destino donde se gestiona el proceso.

Una vez determinado el objetivo del diagnóstico y el ¿qué diagnosticar?, es necesario seleccionar los métodos a aplicar con vista a determinar los logros alcanzados, identificar las dificultades y oportunidades, así como las causas que limitan el logro del objetivo y los elementos que pueden acelerar el cumplimiento del mismo. Los métodos a emplear pueden ser tanto del nivel teórico como empírico y su selección depende fundamentalmente del objetivo propuesto.

Todo lo anterior permitirá sentar las bases y constituye el punto de partida para orientar la mejora o diseño de productos en función de lograr aumentar las utilidades de las empresas mediante la satisfacción de los clientes. Ver fig. 1 


\section{Bibliografía}

Beltrán, Luz C. et al. Diseño y comercialización de productos turísticos. Universidad del Externado. Colombia. 2002.

Funcia, Carlos. Metodología para la mejora o diseño de productos turísticos y su comercialización (MEPROTUR). $1^{\circ}$ Convención internacional de estudios turísticos. Universidad de La Habana. La Habana, Cuba. ISBN No 978-959-16-2015-6. 2010.

Funcia, Carlos \& Plochet García, Reynaldo J. EL qué y el cómo en la aplicación del diagnóstico a las ofertas del destino Cuba. $2^{\circ}$ Convención internacional de estudios turísticos. Universidad de La Habana. La Habana, Cuba. ISBN N 978-959-16-2015-6. 2012.

Kotler, P. et al. Mercadotecnia para hotelería y turismo. Editorial Prentice-Hall Hispanoamericana. México D.F. 1997.

Martín, Ramón. El desarrollo de los productos turísticos: perfeccionamiento de los estudios de concepto y de diseño. Centro de estudios turísticos de La Habana. Cuba. 2003.

Mazur, Glenn H. Despliegue de la función de calidad completa para organizaciones de servicios. Guía de Implementación (QDF). Japón. 2002.

Perelló, José L. Desarrollo y promoción de productos turísticos. Centro de estudios turísticos. Universidad de La Habana. Cuba. 2001.

SECTUR de México. Cómo desarrollar productos turísticos competitivos. México D.F. 1998. 
